\title{
SUBCUTANEOUS MIGRATION OF AN ACCIDENTALLY INGESTED FISHBONE
}

\author{
Rinki Das, Sunilbaran Daschakraborty, Mainak Pal, Deepa Keshavan
}

1. Assistant Professor. Department of General Surgery, Nil Ratan Sircar Medical College, Kolkata.

2. Chief Consultant Gastroenterologist. Department of Medical Gastroenterologist, Ruby General Hospital, Kolkata.

3. RMO-cum Clinical Tutor. Department of General Surgery, SSKM Hospital, Kolkata.

4. Junior Resident. Department of General Surgery, North Bengal Medical College, Darjeeling.

\section{CORRESPONDING AUTHOR:}

Dr. Rinki Das,

11/1/14, Jogipara Road,

Kolkata- 700028.

E-mail: rinkid@ymail.com

ABSTRACT: We report a case of subcutaneous migration of fish bone in a $2 \mathrm{yr}$ old boy presented with a lump in his upper abdomen without any features of peritonitis. The diagnosis could be made only after surgical exploration because of the absence of any clinical suspicion and low specificity of imaging modality. This represents a rare condition with potential complications but a natural tendency of the body to get rid of a foreign material and a favorable outcome after chronic penetration of the gastrointestinal tract by a fish bone.

INTRODUCTION: Gastrointestinal foreign body (GIFB) ingestion either intentional or unintentional is a quite common problem. Type of foreign bodies depends on cultural and dietary habits. Accidentally swallowed fish bone is the commonest ingested foreign body in Asian countries. Commonest group that unintentionally ingests foreign bodies is children, particularly those between ages 6 months and 3 years [1]. Most ingested fish bones commonly get impacted in the upper digestive tract producing symptoms or pass out the gastrointestinal tract silently. However, in a few cases, ingested fish bones can penetrate the gastro intestinal tract and may give rise to life threatening complications because of diagnostic difficulty and delay associated with such conditions. Here we report a case of fish bone ingestion which took an unusual path after penetrating the stomach. However, timely intervention saved the child from any further complication.

CASE REPORT: A 2yr old boy presented with 15 days history of sudden onset vague dull aching continuing upper abdominal pain and a rapidly growing lump at upper abdomen for $1 \mathrm{wk}$. On examination his vitals were stable. Abdominal examination showed localized tender parietal swelling measuring $4 \mathrm{~cm} \times 2 \mathrm{~cm}$ in the epigastric region.

Routine investigations were normal. High resolution ultrasound examination of the parities using a $7.5 \mathrm{MHz}$ probe showed an echogenic $4 \mathrm{~cm} \times 0.33 \mathrm{~cm}$ linear structure at the site of parietal swelling and underlying bowel was found to be stuck to the parietal wall at the same site. However, there was no intra-abdominal free fluid or localized collection.

US finding raised the suspicion of some ingested foreign body because of its close relation to bowel lumen. Therefore upper G I endoscopy was also performed to rule out any foreign body, which was unrevealing. 
Surgical exploration under general anesthesia with endo-tracheal intubation was carried out. A $4 \mathrm{~cm}$ long transverse incision was made on the parietal swelling and the lump was dissected out from the surrounding healthy tissue and in this process a fish bone was revealed which was stuck to the sero-muscular layer of anterior wall of the stomach without any mucosal breach. Sero-muscular defect was repaired. Layers of the abdomen were closed. Post-operative recovery was uneventful. The child was discharged on the fourth post-operative day.

DISCUSSION: Fish bone is the commonest ingested foreign body in Asian countries; particularly in area where custom of eating un-filleted fish is common, accounting to $60-90 \%$ of all ingested foreign bodies [2-4]. This is more common in children either because of missed fish bone during screening of food by the caregiver or because of inexperience of the children when they start self feeding.

Most commonly the ingested fish bones get lodged in the tonsils or the base of the tongue. Other sites of impaction include the vallecula, pyriform fossa, epiglottis, cricopharyngeus, and esophagus. Fish bones which escape this upper digestive tract barrier enter the stomach where the usual fate is acid digestion. However, very thick fish bones and those more than $5 \mathrm{~cm}$ in size generally cannot get digested nor can they pass through the pylorus necessitating endoscopic extraction if symptomatic [5].

In a few cases, ingested fish bones can migrate through GI tract to remote parts of the body and give rise to diagnostic difficulty endangering life. Very rarely, sharp pointed fish bones silently erode the stomach and can migrate in the retroperitoneum. In this process they incite inflammatory response and can mimic gastric or pancreatic neoplasm or they can lead to an inflammatory abscess mimicking pancreatic abscess [6-7]. Cases with acute pancreatitis have also been documented with retroperitoneal migration of fish bone [8]. Fish bone perforation of the gastrointestinal tract is seldom diagnosed preoperatively because of varied presentation and lack of a definite history of fish bone ingestion as these are often ingested accidentally and are forgotten [9].

Radiography is unreliable in the diagnosis of fish bone perforation as the radio-opacity varies among species of the fish and the fish bones are often surrounded by soft tissue masses and edema obscuring the calcium content of the fish bone. Even, in cases where fish bones penetrate any hollow abdominal viscera, free gas under the diaphragm is almost never seen. Usually there is progressive erosion of the gut wall by the fish bone and the defect gradually gets sealed off by fibrin, omentum \& bowel wall. Plain radiograph has sensitivity and specificity of $39 \%$ and $72 \%$, respectively for detection of fish bone $[8,10]$.

Upper GI endoscopy is very helpful as it may be both diagnostic and therapeutic. USG is less reliable. CT scan is a superior investigation and helps in detection of location, determination of size of the fish bone as well as its relation to the vital organs [8].

In our case, lack of suspicion because of absence of a definite history and very rare occurrence of such incidences failed us to make a pre operative diagnosis of fish bone migration. Nevertheless, retrospective review of the ultrasound film revealed a linear pattern of calcification consistent with a fish bone.

To our knowledge, this is the first case report of trans gastric migration of fish bone into subcutaneous tissue following ingestion in a child. Although current guidelines of therapy for fish bone ingestion in children remains conservative, this case illustrates the possibility of unpredictable complications which necessitate surgical intervention. 


\section{REFERENCES:}

1. Chen MK, Beierle EA: Gastrointestinal foreign bodies. Pediatr Ann 2001; 30:736-42

2. Zhang S, Cui Y, Gong X, Gu F, Chen M, Zhong B. Endoscopic management of foreign bodies in the upper gastrointestinal tract in South China: a retrospective study of 561 cases. Dig Dis Sci. 2010 May; 55(5):1305-12

3. Wai Pak M, Chung Lee W, Kwok Fung H, van Hasselt CA. A prospective study of foreignbody ingestion in 311 children. Int J Pediatr Otorhinolaryngol 2001 Apr 6;58(1):37-45

4. Arana A, Hauser B, Hachimi-Idrissi S, Vandenplas Y.Management of ingested foreign bodies in childhood and review of the literature. Eur J Pediatr 2001; 160: 468-472

5. Koch H, Operative Endoscopy. Gastrointest endosc, 1977; 24:65-68.

6. A. Bajwa, H. Seth and F. Hughes. Ingested fishbone mimicking a gastric submucosal tumour, Grand Rounds Vol 7 pages 42-44

7. Goh BK, Jeyaraj PR, Chan HS, et al. A case of fish bone perforation of stomach mimicking a locally advanced pancreatic carcinoma. Dig Dis Sci 2004; 49: 1935-1937.

8. Brian K. P. Goh, Yu-Meng Tan Shueh-En Lin, Pierce K. H. Chow, Foong-Koon Cheah, London L. P. J. Ooi1, Wai-Keong Wong. CT in the Preoperative Diagnosis of Fish Bone Perforation of the Gastrointestinal Tract. AJR 2006; 187:710-714 0361-803X/06/1873710

9. Sheng-Der Hsu, De-Chuan Chan, Yao-Chi Liu. Small-bowel perforation caused by fish bone. World J Gastroenterol 2005;11(12):1884-1885

10. Lue AJ, Fang WD, Manolidis S. Use of plain radiography and computed tomography to identify fish bone foreign bodies. Otolaryngol Head Neck Surg 2000; 123: 435-8.

\section{High-resolution ultrasound of the parities reveals an elongated foreign body in the} subcutaneous tissue of the abdomen in close relation to bowel.

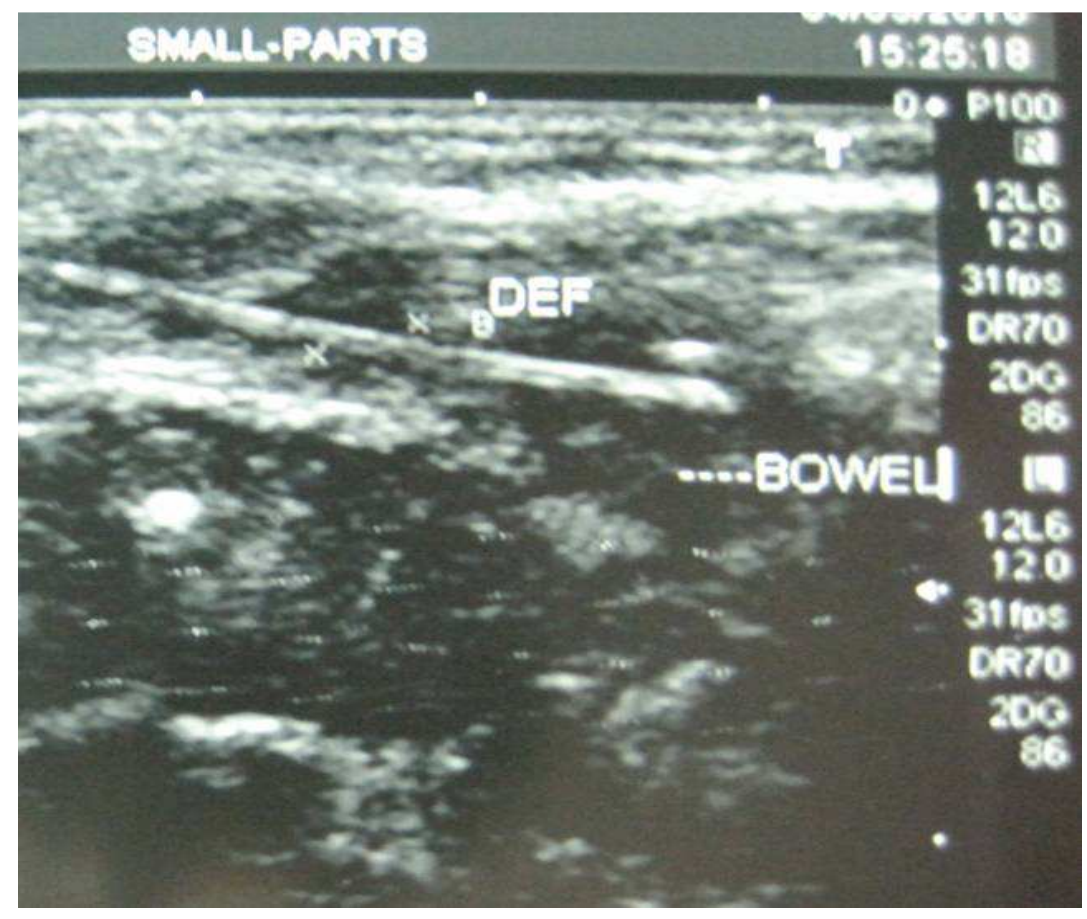




\section{CASE REPORT}

Fig.2: The fishbone revealed during surgical exploration of the parietal lump

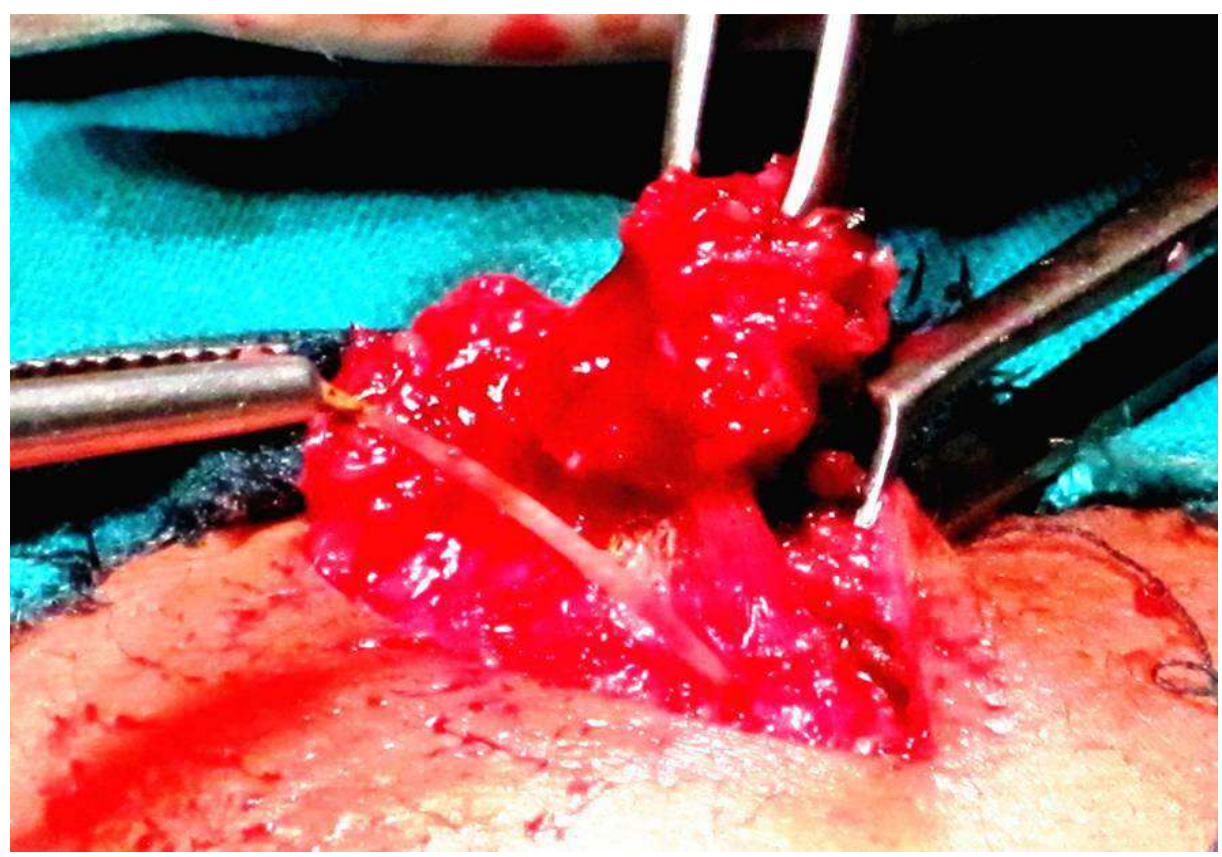

Fig. 3: Excised lump and the fish bone



